\title{
Design and Testing of a MEMS Acoustic Emission Sensor System
}

\author{
David W. Greve ${ }^{\mathrm{a}}$, Irving J. Oppenheim ${ }^{1 \mathrm{~b}}$, Amelia P. Wright ${ }^{\mathrm{b}}$, and Wei Wu ${ }^{\mathrm{a}}$ \\ ${ }^{a}$ Dept. of Electrical and Computer Engineering, Carnegie Mellon University, Pittsburgh, PA 15213 \\ ${ }^{\mathrm{b}}$ Dept. of Civil and Environmental Engineering, Carnegie Mellon University, Pittsburgh, PA 15213
}

\begin{abstract}
We present four new findings pertaining to MEMS sensors for acoustic emission detection. Our sensors are resonanttype capacitive transducers, operating with a frequency between $100 \mathrm{kHz}$ and $500 \mathrm{kHz}$, fabricated in the PolyMUMPS process. The sensitivity of a resonant transducer is related to the sharpness of its resonance, measured by the quality factor Q, and operating in a coarse vacuum will increase Q. We describe a practical laboratory method for sealing and evacuating our MEMS sensor, and present measurements showing Q in the evacuated packages to be 2.4 to 3.6 times greater than under atmospheric pressure. We also describe our theoretical analysis of noise sources in the electromechanical behavior of a resonant, capacitive-type transducer sensitive to out-of-plane motion, with particular interest in noise resulting from mechanical excitation of the moving plate by air molecule impact. We report on a new transducer design to sense out-of-plane motion featuring a moving plate constructed as an open grill rather than as a plate perforated by etch holes. Characterization measurements show the open grill design to have a higher Q than a comparable perforated plate transducer. Finally, we report on another novel transducer designed to sense in-plane motion. The sensor is a comb finger capacitive transducer, and theoretical predications predict the in-plane sensor to have a much higher $\mathrm{Q}$ than the out-of-plane sensors. We show experimental measurements confirming these design characteristics, and we show results from pencil lead break experiments.
\end{abstract}

Keywords: acoustic emissions, MEMS, damping, transducer sensitivity, in-plane sensing

\section{INTRODUCTION}

Acoustic emission (AE) testing is a nondestructive evaluation method used to monitor civil infrastructure such as steel bridges and pressure vessels. An acoustic emission is a transient ultrasonic wave released from microscopic zones during plastic deformation. Civil structures contain flaws, such as fatigue cracks that grow during cyclic loading and can approach critical fracture lengths. Detection of AE events in a structure is evidence of an active or growing flaw. Conventional AE sensors consist of piezoelectric transducers, however our research group uses MEMS fabrication to develop capacitive resonant-type transducers made up of an array of independent transducers on a small chip. The behavior of these devices and their use as acoustic emission detectors is described in a paper by Ozevin et al. [1]. In this paper, we present a simple laboratory method to seal one of our MEMS devices in a coarse vacuum and demonstrate an increase in sensitivity using characterization measurements. We also present a theoretical analysis of noise sources in our MEMS device that predicts noise levels consistent with measured noise levels. In addition, we describe a new transducer design featuring an open grill design and present characterization measurements showing an increase in sensitivity over previous designs. Conventional piezoelectric AE sensors and MEMS AE sensors built to date detect displacements normal to the contact surface (in the out-of-plane direction). However, obtaining a three-dimensional displacement history (in the out-of-plane and in-plane directions) will provide a rich and informative signal and will be an advance in $\mathrm{AE}$ sensing. In this paper, we describe the design of a new capacitive resonant-type transducer sensitive to in-plane motion accompanied by characterization measurements.

\footnotetext{
${ }^{1}$ Contact author: ijo@cmu.edu; 412-268-2950; Carnegie Mellon University, Department of Civil and Environmental Engineering, Pittsburgh, PA, 15213.
} 


\section{PERFORATED PLATE TRANSDUCER FOR OUT-OF-PLANE SENSING}

Figure 1 shows a CAD layout of a $5 \times 5 \mathrm{~mm}$ MEMS chip containing four independent perforated plate transducers for out-of-plane sensing, with design frequencies between 126 and $500 \mathrm{kHz}$. Figure 2a shows a completed four-channel AE sensing system $[2,3]$. Each of the four transducers is made up of an array of units, and each unit is a perforated plate suspended by four flexural springs, shown in Figure $2 \mathrm{~b}$. Each unit is $125 \mu \mathrm{m}$ on each side with $3.5 \mu \mathrm{m}$ square etch holes on a triangular grid with $8 \mu \mathrm{m}$ spacing between holes. The perforated plate is supported by four flexural springs with a $1.25 \mu \mathrm{m}$ gap between the moving plate and stationary electrode below. FEM simulations were used to select spring lengths in order to achieve target design frequencies between 126 and $500 \mathrm{kHz}$. Each of the four transducers consists of 144 units in a $12 \times 12$ array to obtain a target design capacitance near $13 \mathrm{pF}$. The MEMS chip is mounted in a Spectrum Semiconductor Materials CPG06856 pin-grid array ceramic package, $26 \times 26 \mathrm{~mm}$, chosen because its smooth bottom surface will provide good acoustic coupling to structural plates. The ceramic package attaches to the bottom PC board and a Sullins $2.0 \mathrm{~mm}$ connector attaches the bottom board to the top PC board containing four amplifier circuits, each with a nominal gain of $100 \mathrm{~V} / \mathrm{V}$. The volume of the system is $26 \times 26 \times 15 \mathrm{~mm}$ excluding the DB9 connector.

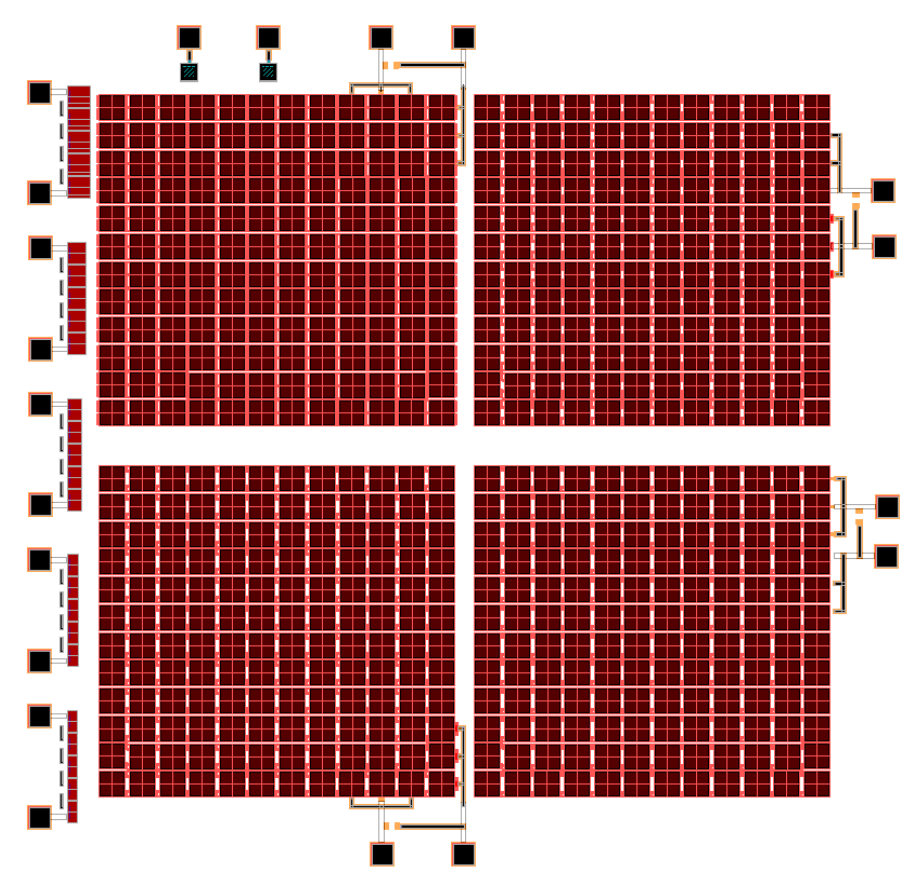

Figure 1. CAD layout of $5 \times 5 \mathrm{~mm}$ MEMS chip containing four perforated plate transducers 

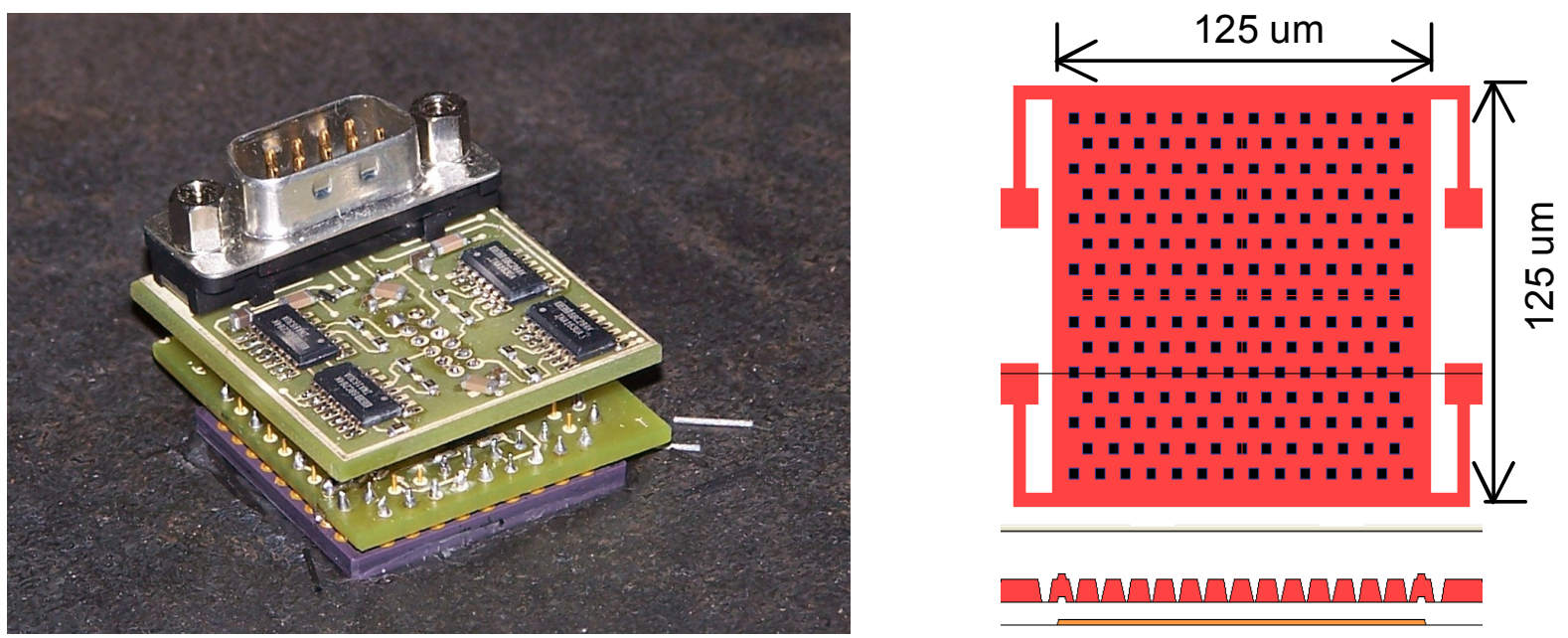

Figure 2. (a) Four-channel AE sensor system in $35 \times 35 \times 30 \mathrm{~mm}$ volume; (b) CAD layout and section of one unit.

The sensitivity of this transducer is related to the sharpness of its resonance peak and is commonly quantified by the quality factor $Q$. The damping effect of air is one of the dominant influences on $Q$. Damping occurs both as acoustic radiation into the air and as squeeze film damping, as air is forced through the gap between the moving and stationary plate. Operation in a coarse vacuum would reduce the damping effect of air and increase $Q$, and we have developed a laboratory method to seal and evacuate the device within its package. The CPG06856 package has an accompanying lid, plated with nickel and gold. In our method, a small hole is drilled in the lid, the perimeter of the lid is soldered to the CPG06856 package, and a small amount of solder is place near the hole without covering it. The package is then placed in a vacuum chamber containing a soldering iron, which can translate on a vertical axis. After the chamber is evacuated and the soldering iron is heated, the iron is lowered to melt the solder over the hole. The soldering iron is raised allowing the solder to cool and seal the package. Once the solder is completely cooled, the package can be removed from the chamber. This method has proven reliable and the seal appears to be durable.

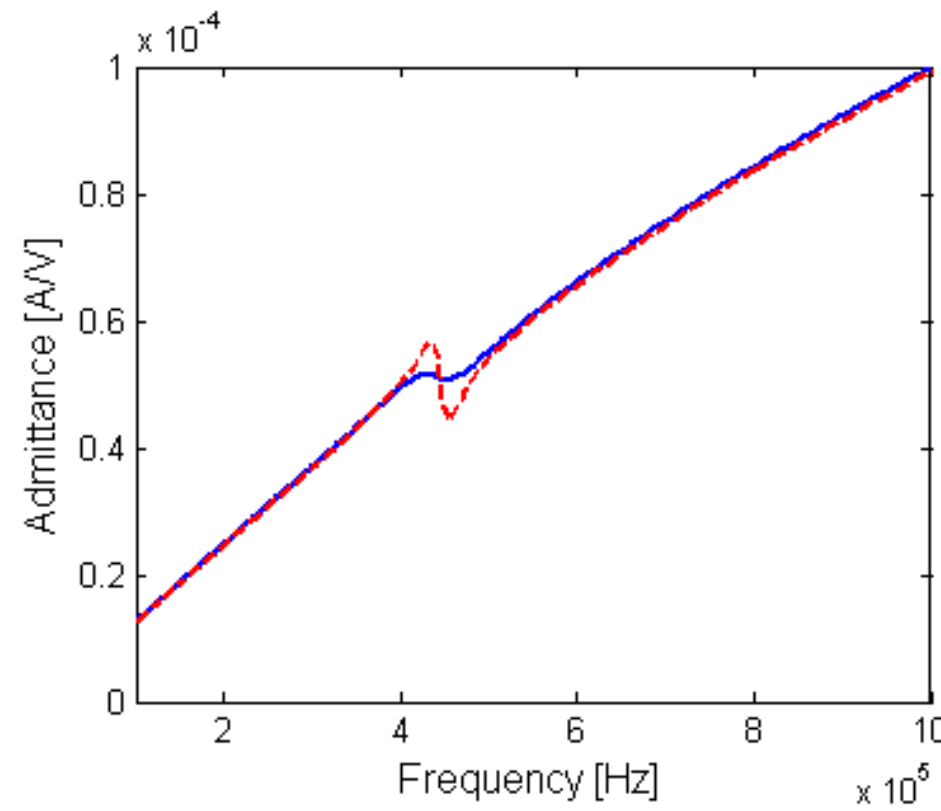

Figure 3. Admittance at resonance, $f=443 \mathrm{kHz}, Q=5.5$ (atmospheric), $Q=19.7$ (evacuated) 
Figure 3 shows measured admittance magnitude as a function of frequency for a transducer with a design frequency of $500 \mathrm{kHz}$, and a measured resonance near $443 \mathrm{kHz}$, both before and after evacuation. This measurement was performed with a DC bias of $9 \mathrm{~V}$. The broader resonance (solid line) is the admittance plot at atmospheric pressure, corresponding to a $Q$ of 5.5, while the sharper resonance (dashed line) is the admittance plot performed after evacuation, corresponding to a $Q$ of 19.7. The measured admittance of the sealed sensor is unchanged after 28 days, suggesting a very low leak rate. The increase in $Q$ is approximately fourfold and improves the sensitivity of the transducer. However, the $Q$ value is significantly lower than that typically observed by other researchers who have built resonators in the PolyMUMPS process with a measured $Q$ value between 300 and 550 [4]. We do not think this relatively low $\mathrm{Q}$ can be attributed to incoherent excitation of the transducers; the $12 \times 12$ array occupies an area less than $2 \mathrm{~mm}$ on a side, which is small compared to the ultrasonic wavelength in steel at the frequencies of our transducers. It is more likely that the 144 units do not have identical resonant frequencies, and that the observed $Q$ is limited by imperfect coherence in their response. For example, the anchor stiffness will differ from the perimeter of the array to the interior, creating one source for slight deviations in the resonant frequencies. Another likely source is variation in the spring constant due to manufacturing variation in the polysilicon beam width. The effect of imperfect coherence would be a "spreading" of the aggregated peak, which appears as a broader peak in admittance measurements, resulting in a lower $Q$.

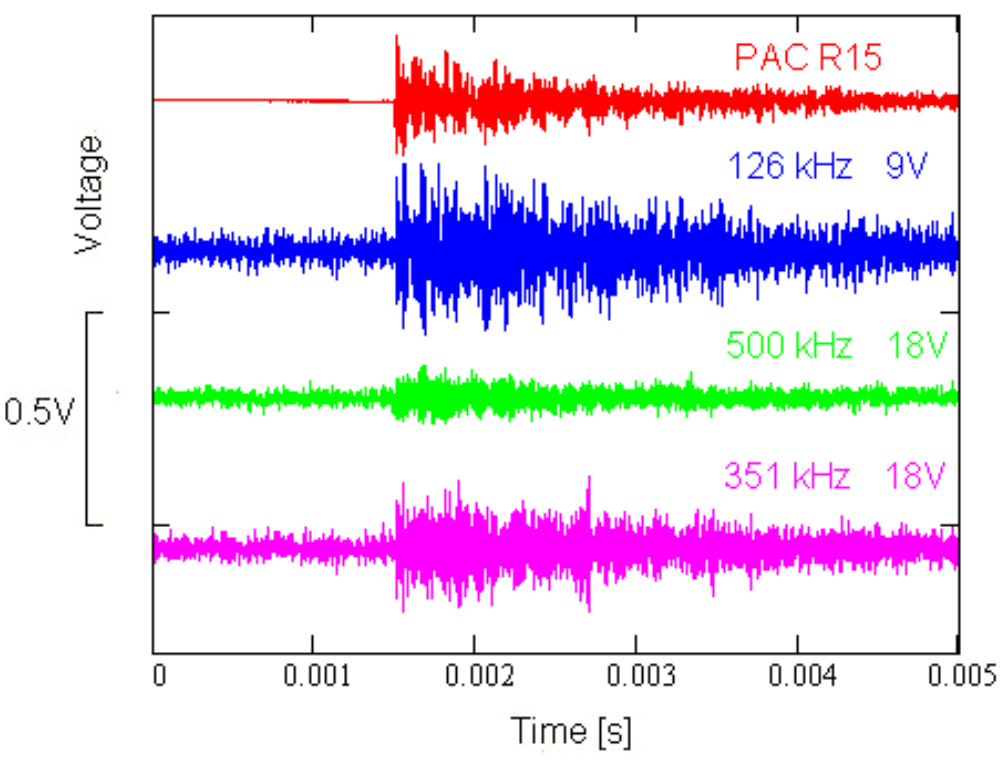

Figure 4. Pencil lead break response, comparing three MEMS sensors to PAC R15 sensor

We applied pencil lead breaks on a large steel plate, $9.5 \mathrm{~mm}$ thick, with the MEMS system (Figure 2a) and a PAC R15 sensor mounted symmetrically $20 \mathrm{~mm}$ from the source. Figure 4 shows the response of three channels of the MEMS device, after onboard amplification, and the raw response of the PAC R15 sensors. For each MEMS channel, we indicate its design frequency and bias voltage in Figure 4. Signal strength from the sensor channels on the MEMS device is comparable to that from the PAC R15 sensor, although the signal-to-noise ratio is not as favorable.

We recently developed a theoretical analysis of noise sources [5] in the electromechanical behavior of a resonant, capacitive-type transducer. We determine that Brownian noise from impact excitation of the moving plate by air molecules, as examined by Gabrielson [6], is the most significant noise source. Summarizing those findings here, we report the RMS amplitude of the current resulting from such agitation to be

$$
i_{R M S}=\frac{V_{D C} C_{0}}{g} \sqrt{4 k_{B} T} \cdot\left[\sqrt{m \omega_{0}} \int f(\omega, Q) d \omega\right]
$$

where $V_{D C}$ is the DC bias voltage, $g$ is the gap between the capacitor plates, $C_{0}$ is the capacitance at rest, $k_{B}$ is the Boltzmann constant, $T$ is the temperature $\left({ }^{\circ} \mathrm{K}\right), \omega_{0}$ is the circular resonant frequency, and $m$ is the mass of the spring- 
supported plate. The term in square brackets, which includes an integral over frequency, captures the dynamic response of the resonator to air molecule impact. The integral depends upon the quality factor $Q$, together with the influence of amplifier bandwidth; the reader is referred elsewhere [5] for the derivation and details. However, if the amplifier bandwidth $\omega_{\mathrm{H}}$ encloses the resonant peak, during contour integration (not shown) the upper limit of the integral can be extended to infinity. In this case, the result,

$$
i_{R M S}^{2}=\left(\frac{V_{D C} C_{0}}{g}\right)^{2} \frac{k_{B} T}{m},
$$

is almost independent of Q. Recent measurements over a wide range of design resonant frequencies (from 126 to 500 $\mathrm{kHz}$ ) show reasonable comparison between the predicted and measured noise level [5].

\section{OPEN GRILL TRANSDUCER FOR OUT-OF-PLANE SENSING}

We report on a new out-of-plane transducer design featuring a moving plate constructed as an open grill rather than as a plate with periodically spaced etch holes. Figure 5a shows the $5 \times 5 \mathrm{~mm}$ MEMS chip on which this transducer is fabricated. This MEMS chip also includes two additional out-of-plane transducers and a transducer sensitive to in-plane motion, which will be discussed in the next section. Figure 5b shows a CAD layout of a single unit, featuring the open grill supported by four flexural springs. The beams forming the grill are $3 \mu \mathrm{m}$ in width, with $3 \mu \mathrm{m}$ spacing, and a gap of $1.25 \mu \mathrm{m}$ between the open grill and the stationary electrode below. The outside dimensions of the open grill are $138 \times$ $140 \mu \mathrm{m}$. FEM simulation was used in selecting the spring length to achieve a target design frequency of $250 \mathrm{kHz}$. The transducer consists of a $9 \times 8$ array of units to achieve a design capacitance, $C_{0}$, of $5.8 \mathrm{pF}$, calculated based on the net area of the open grill.
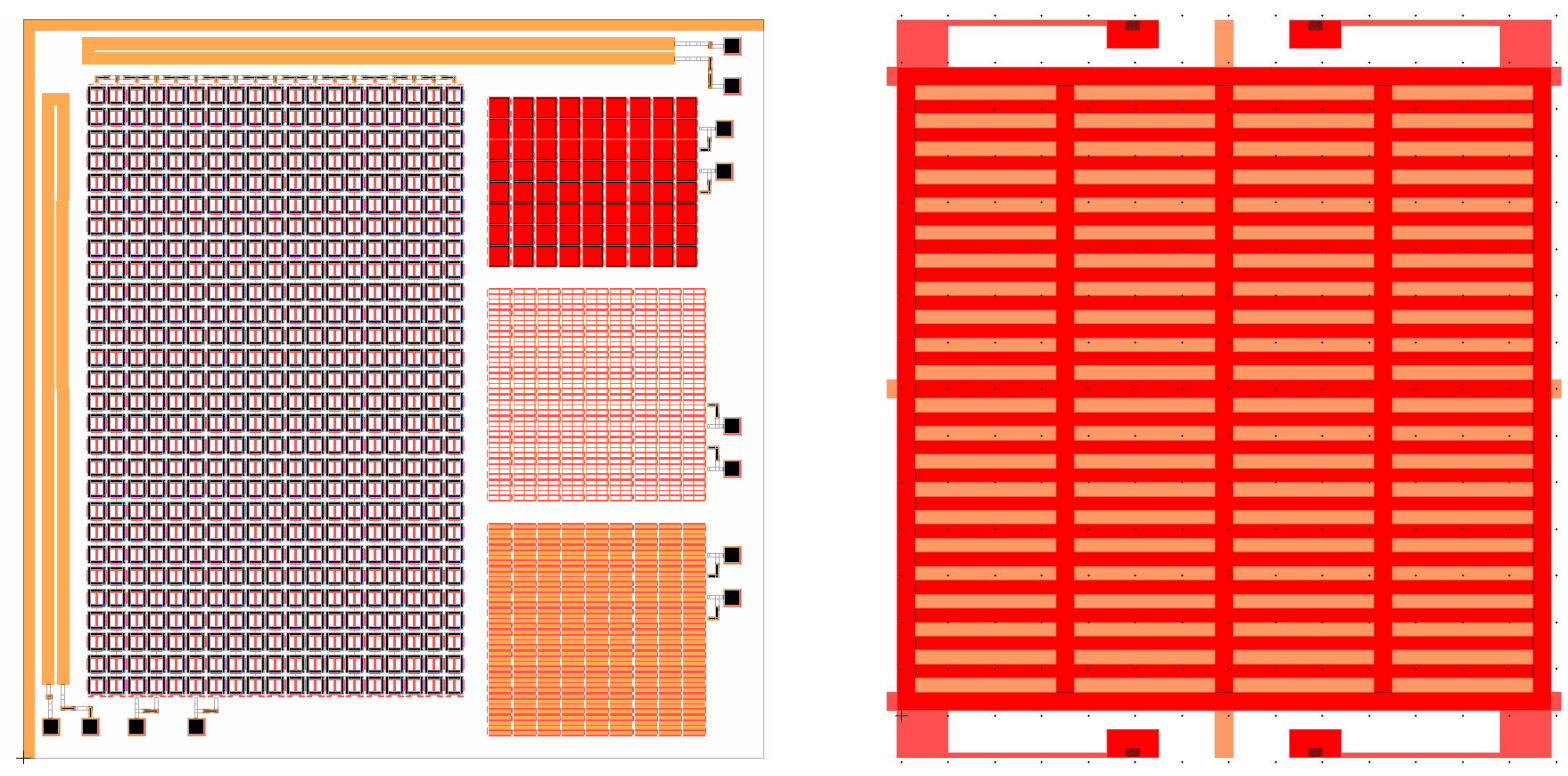

Figure 5. (a) CAD layout of one $5 \times 5 \mathrm{~mm}$ MEMS chip with three out-of-plane transducers (right side) and one in-plane transducer (left side); (b) CAD layout of open grill design for out-of-plane sensing

A capacitive spring-mass system such as this should show an increase in capacitance with applied DC voltage because the attractive electrostatic force induced by the DC voltage will cause the spring-suspended plate to deflect towards the stationary electrode, increasing the capacitance between the two plates. Figure 6a shows a plot of capacitance versus voltage $(C-V)$ confirming the expected behavior, but showing a capacitance at $0 \mathrm{~V}, C_{0}$, near $10.9 \mathrm{pF}$. Subsequent FEM simulations confirm the measured capacitance, $C_{0}$, and show that $C_{0}$ approximates that of the gross area of a whole plate 
rather than the net area of the open grill due to electric field fringing. In other words, the "cutouts" between the beams of the open grill do not diminish the capacitance.
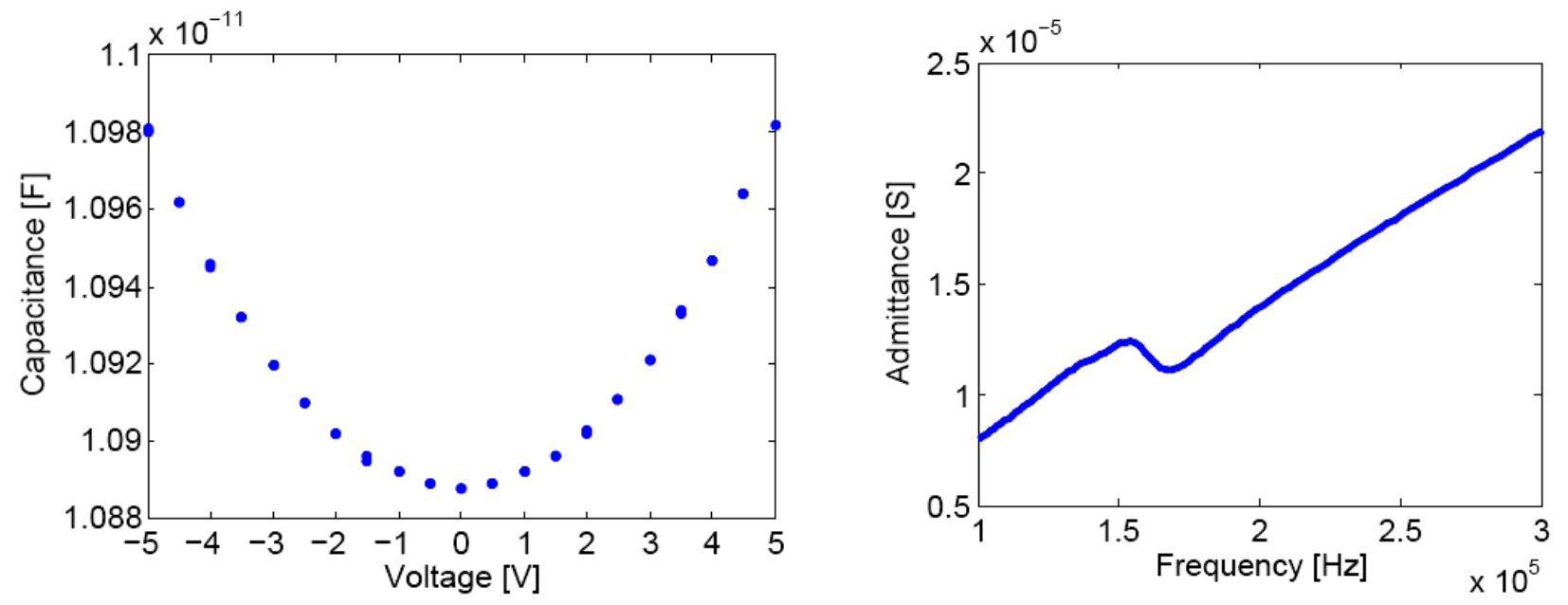

Figure 6. (a) $C$ - $V$ plot showing $C_{0}=10.9 \mathrm{pF}$; (b) Admittance at resonance, $f=160 \mathrm{kHz}, Q=6.7$

Figure $6 \mathrm{~b}$ shows an admittance plot near resonance, which is observed to be $160 \mathrm{kHz}$, corresponding to a $Q$ of 6.7 . When comparing $Q$ for a perforated plate transducer with that of the open grill transducer, both devices must have comparable resonant frequencies, because radiation and squeeze-film damping will vary with frequency. A perforated plate transducer (such as Figure $2 \mathrm{~b}$ ) with a resonant frequency of $182 \mathrm{kHz}$, reasonably close for purposes of comparison, displayed a $Q$ near 2.0; based upon this comparison, the open grill design shows substantial improvement in $Q$. Compared to a perforated plate, we predicted that the open grill geometry allows freer venting of air between plates, reducing squeeze-film damping, and increasing $Q$. We interpret these results as evidence qualitatively confirming that prediction.

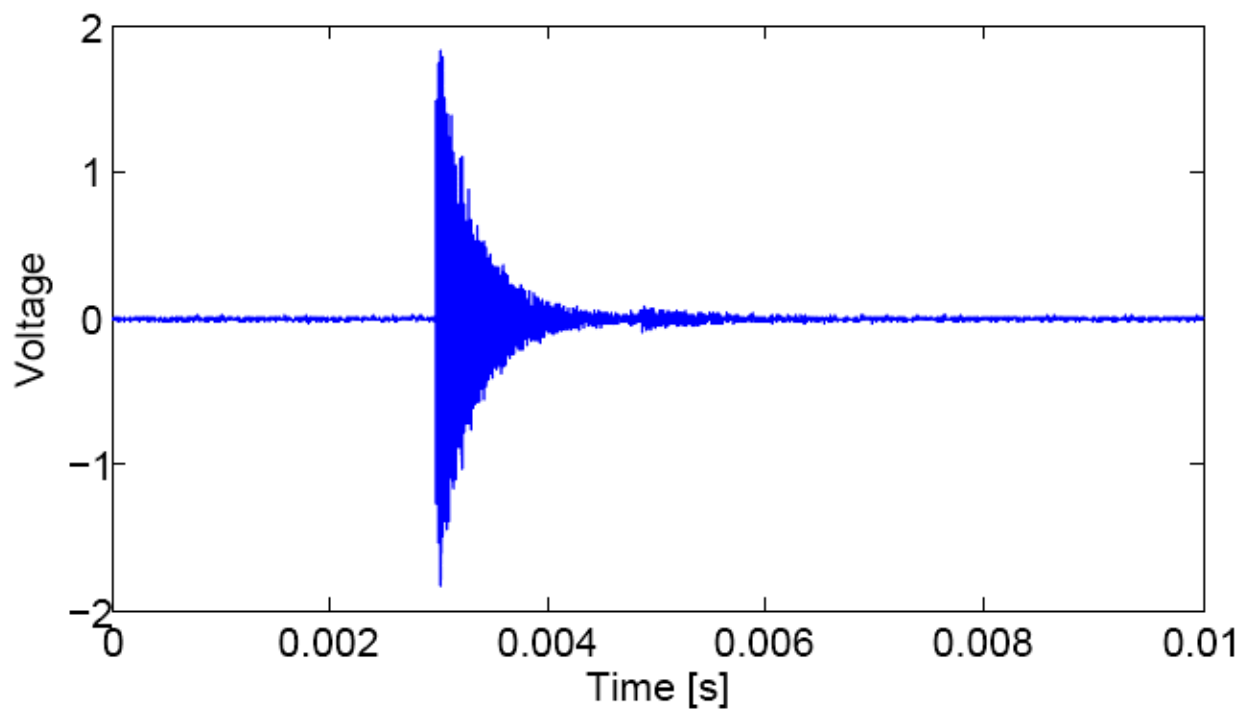

Figure 7. Pencil-lead break response of open grill out-of-plane sensor 
The open grill out-of-plane transducer is mounted in an identical ceramic package and has the same amplification system as the perforated plate transducer shown in Figure 2a. Figure 7 depicts the characteristic response of the open grill outof-plane sensor to a pencil-lead break applied directly to the ceramic package.

\section{FINGER TRANSDUCER FOR IN-PLANE SENSING}

Finally, we report on another new transducer designed to detect in-plane motion. This device is a finger-type capacitive transducer with a design frequency of $250 \mathrm{kHz}$. Figure 8 shows a CAD layout of a single unit, showing a stationary spine with projecting fingers, which form capacitors with fingers projecting from the thick frame, which is attached to four flexural springs. Each unit is $128 \times 132 \mu \mathrm{m}$, and again, FEM simulation was used to select the flexural spring length to achieve the target design frequency. The pitch between beams is unsymmetrical in order to effect a change in capacitance during motion. The transducer consists of 532 units in a $19 \times 28$ array to obtain a target design capacitance, $C_{0}$, of $3.1 \mathrm{pF}$. The predicted $Q$ for this device is significantly higher (predicted damping is much lower) than for the outof-plane sensors, because in-plane motion produces a direct shearing of the air gap, rather than a squeeze-film or radiation actuation of the air. In this device, the damping that occurs during out-of-plane motion (squeeze-film and radiation damping) is significantly higher than damping that occurs during in-plane motion (shearing). This difference effectively isolates the desired in-plane mechanical response from the unwanted out-of-plane response.

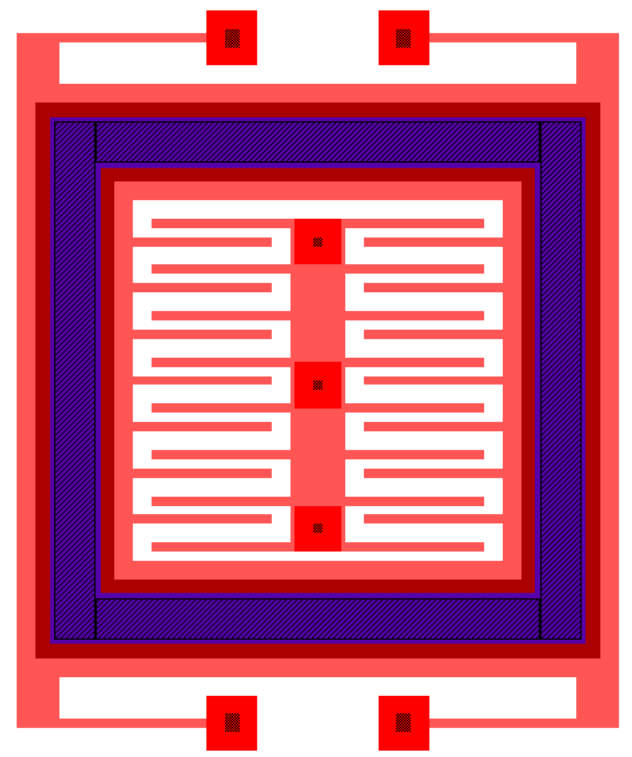

Figure 8. CAD layout of finger-type transducer designed to detect in-plane motion

Figure 9a shows the $C$ - $V$ plot, confirming the expected behavior of a capacitive spring-mass system, with a measured $C_{0}$ value of $2.95 \mathrm{pF}$. Figure $9 \mathrm{~b}$ shows admittance measurements near the resonant frequency, observed to occur near 176 $\mathrm{kHz}$, corresponding to a $Q$ value of 197 . These characterization measurements confirm the design characteristics outlined above, and suggest that the transducer may be an effective way to sense in-plane particle motion. In principle, a MEMS chip can be fabricated with one transducer sensitive to in-plane motion, a second identical in-plane transducer orthogonal to the first one, and a third transducer sensitive to out-of-plane motion to create a complete sensor system sensitive to the three dimensions of particle motion. 

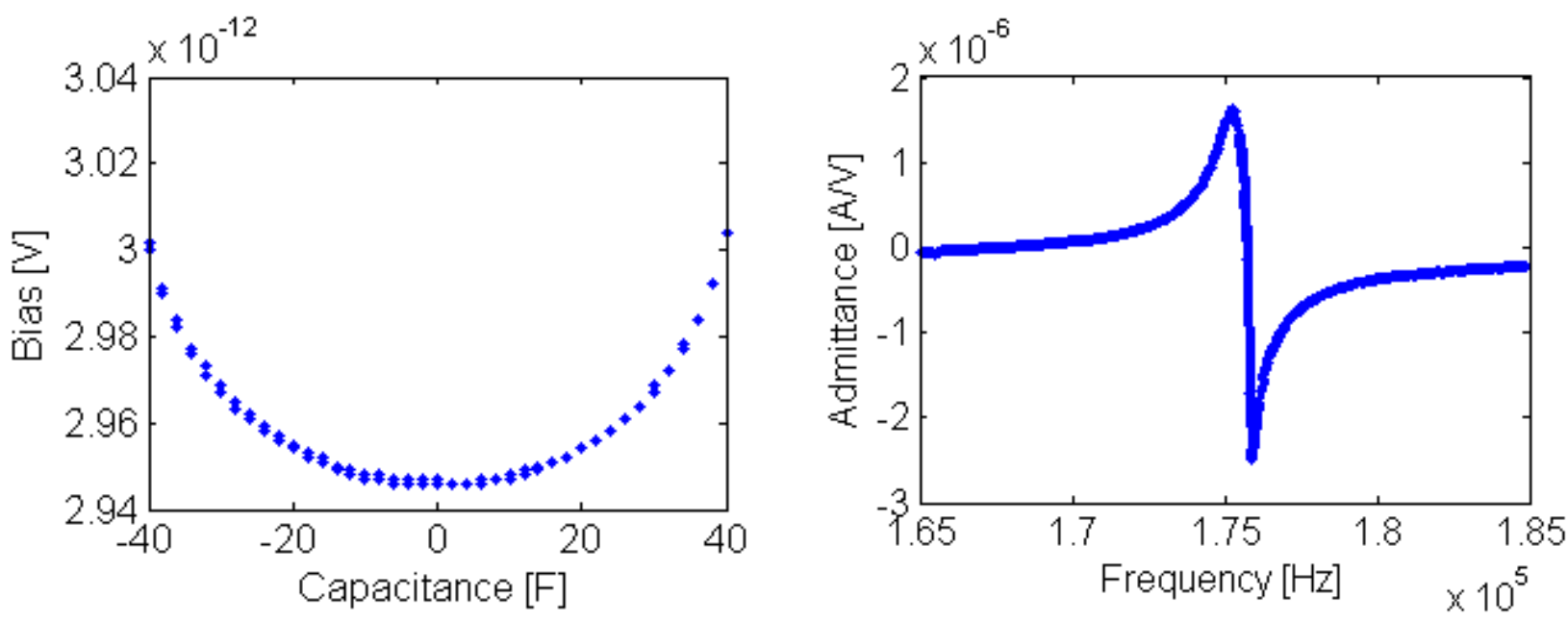

Figure 9. (a) $C-V$ plot showing $C_{0}=2.95 \mathrm{pF}$; (b) Admittance at resonance, $f=176 \mathrm{kHz}, Q=197$

The in-plane transducer is mounted in an identical ceramic package and has the same amplification system as the out-ofplane transducers shown in Figure 2a. Figure 10 shows the response of the in-plane sensor to a pencil-lead break applied directly to the ceramic package. A beat frequency is observed, near $8 \mathrm{kHz}$, which is consistent with a slight difference in frequency between interior and exterior units in the $19 \times 28$ array. This difference in frequency can be explained by a small difference in the length of the flexural springs on the perimeter. The beat effect is only visible because the inplane device is a high- $Q$ resonator.

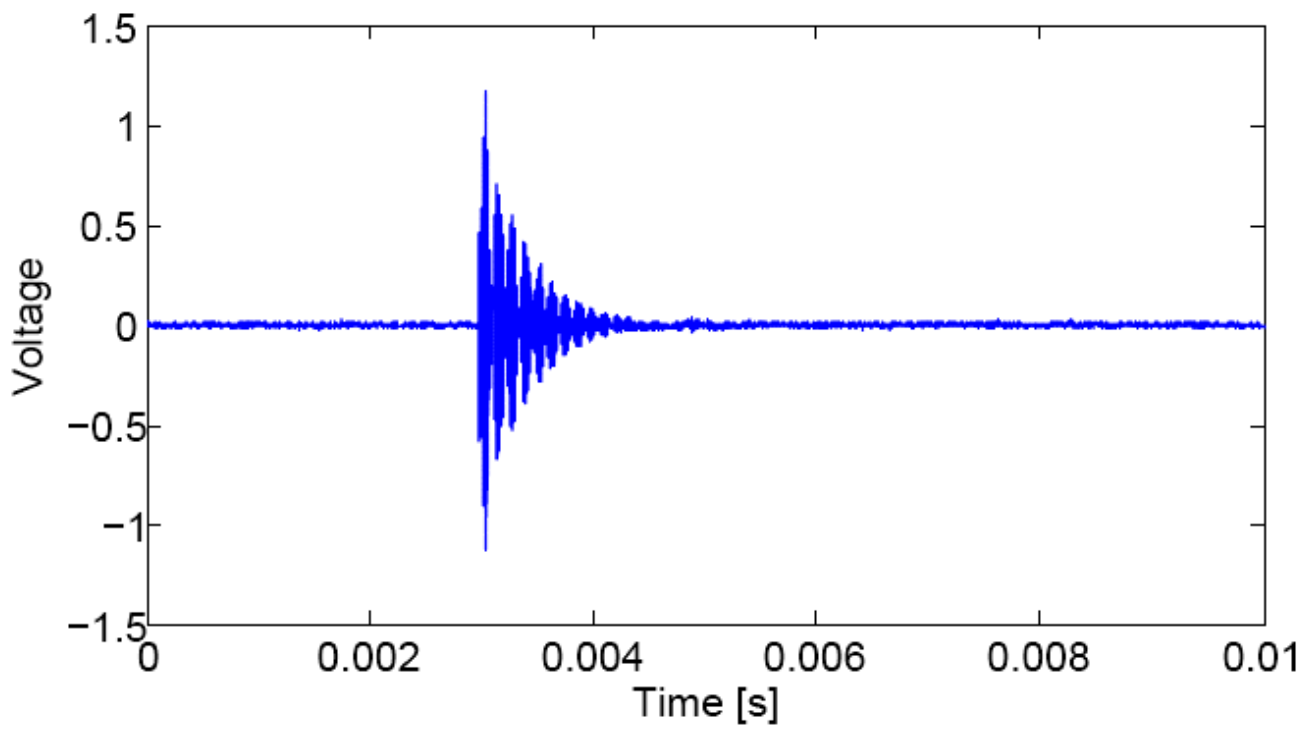

Figure 10. Pencil-lead break response of in-plane sensor

\section{DISCUSSION AND CONCLUSIONS}

Our research group has developed resonant-type capacitive MEMS sensors for acoustic emission detection, with frequencies between 100 and $500 \mathrm{kHz}$. The damping effect of air limits the sensitivity of these devices, as measured as the quality factor $Q$, and noise limits the effective sensitivity as measured by the SNR. We seek to increase the $Q$ value of our devices and have presented four recent advances in our understanding of the mechanics of these sensors related to 
improving sensitivity. We have shown an effective laboratory method for vacuum sealing with a low leak rate that improves device sensitivity approximately fourfold. The response of our vacuum-sealed sensor to a pencil-lead break is comparable to a commercial transducer, although the SNR is not as favorable. Our theoretical noise analysis identifies Brownian motion and the impact of air molecules with the resonator as the major source of noise and predicts the noise level to be independent of $Q$. Experimental measurements confirm our predicted noise levels and suggest that increasing $Q$ increases the signal-to-noise ratio. We have also reported on a new design featuring an open grill rather than a perforated plate as the moving (spring-suspended) electrode of our capacitive transducer. This open grill design decreases the squeeze-film damping between the moving plate and stationary electrode. Finally, we described a new sensor designed to detect in-plane particle motion. Characterization measurements confirm that it is minimally damped because in-plane motion produces only a shearing of the air gap rather than squeeze-film or radiation damping. Our research findings suggest that a complete sensor system for three-dimensional sensing can be fabricated by collocating two in-plane sensors orthogonal to each other and one out-of-plane sensor on a single MEMS chip.

\section{ACKNOWLEDGEMENTS}

This work is supported by the National Science Foundation, Grant CMS-0329880 entitled SENSORS: Collaborative Research: MEMS for Multi-Mode Civil Infrastructure Sensing, and by the Pennsylvania Infrastructure Technology Alliance. We gratefully acknowledge contributions of our research collaborators at Lehigh University, Professor S. P. Pessiki and Dr. D. Ozevin, who is now a Research Scientist with Physical Acoustics Corporation. The opinions, findings, conclusions, and recommendations expressed in this material are those of the current authors and do not necessarily reflect the views of the sponsors or of our collaborators.

\section{REFERENCES}

1. Ozevin, D., Greve, D. W., Oppenheim, I. J., and Pessiki, S. P., "Resonant capacitive MEMS acoustic emission transducer," Smart Materials and Structures, 15(6), 1863-1871 (2006).

2. Greve, D. W., Oppenheim, I. J., and Wu, W., "Modes and damping in cmut transducers for acoustic emissions," IEEE Ultrasonics Conf., 661-664 (2006).

3. Greve, D. W., Oppenheim, I. J., Wu, W., and Wright, A. P., "Development of a MEMS acoustic emission sensor system," Proc. of SPIE 6529 (2007).

4. Bahreyni, B. and Shafai, C., "Fabrication of piezoresistive sensors in standard MEMS foundry processes," IEEE Sensors Conf., 215-218 (2005).

5. Wu, W., Greve, D. W., and Oppenheim, I. J., "Characterization and Noise Analysis of Capacitive MEMS Acoustic Emission Transdcuers," IEEE Sensors Conf., 1152-1155(2007).

6. Gabrielson, T. B., "Mechanical-thermal noise in micromachined acoustic and vibration sensors," IEEE Trans. Electron. Devices, 40(5), 903-909 (1993). 